\title{
Leveraging Medical Literature for Section Prediction in Electronic Health Records
}

\author{
Sara Rosenthal \\ IBM Research \\ Yorktown Heights, NY, USA \\ sjrosenthal@us.ibm.com
}

\author{
Ken Barker \\ IBM Research \\ Yorktown Heights, NY, USA \\ kjbarker@us.ibm.com
}

\author{
Jason Zhicheng Liang* \\ Rensselaer Polytechnic Institute \\ Troy, New York, USA \\ liangz 4 erpi.edu
}

\begin{abstract}
Electronic Health Records (EHRs) contain both structured content and unstructured (text) content about a patient's medical history. In the unstructured text parts, there are common sections such as Assessment and Plan, Social History, and Medications. These sections help physicians find information easily and can be used by an information retrieval system to return specific information sought by a user. However, it is common that the exact format of sections in a particular EHR does not adhere to known patterns. Therefore, being able to predict sections and headers in EHRs automatically is beneficial to physicians. Prior approaches in EHR section prediction have only used text data from EHRs and have required significant manual annotation. We propose using sections from medical literature (e.g., textbooks, journals, web content) that contain content similar to that found in EHR sections. Our approach uses data from a different kind of source where labels are provided without the need of a timeconsuming annotation effort. We use this data to train two models: an RNN and a BERTbased model. We apply the learned models along with source data via transfer learning to predict sections in EHRs. Our results show that medical literature can provide helpful supervision signal for this classification task.
\end{abstract}

\section{Introduction}

Electronic Health Records (EHRs) are an important tool used by physicians that contain an abundance of information about each patient. In addition to aiding the physician in providing patient care, EHRs can be used for downstream tasks

\footnotetext{
${ }^{*}$ Work completed during internship at IBM Research
}

such as predicting patient outcome, representation learning, and information extraction (Shickel et al., 2017). All of these tasks can be applied to the unstructured text parts of an EHR. Furthermore, they would benefit from the section structure to pinpoint the likely location where the information should be found (e.g. drug codes are more likely to be in the Medication section). However, when physicians edit the unstructured text parts of an EHR, there are no set rules for indicating sections and format is not strictly enforced, nor is there any markup (e.g. XML). An added difficulty is that the formatting is not uniform across EHRs. This is even more common when the EHRs come from different hospitals. For example, one EHR may have the section heading "Assessment and Plan" where another may use "A\&P". As an added challenge there may also be nested sections, such as information about a specific medication. Being able to predict sections and headers in EHRs automatically is beneficial to physicians. It allows them to find information easily as well as discover errors and omissions in an EHR.

Consider the publicly available EHR note ${ }^{1}$ in Figure 1. The note is divided into the 10 sections found in that EHR (Problems, Medications, History, etc.). This example provides insight into why section prediction can be a difficult task. Although most of the headers appear to be bold, there is also plenty of bold text which is not the main header (e.g. see the History section). Additionally, in some cases (e.g. Allergies section) there is no text under the header at all. This makes it difficult to segment the data appropriately. Finally, although

\footnotetext{
1http://www.ahrq.gov/professionals/ prevention-chronic-care/improve/system/ pfhandbook/mod8appbmonicalatte.html
} 
Problems

DIABETES MELLITUS (ICD-250.)

HYPERTENSION, BENIGN ESSENTIAL (ICD-401.1)

\section{Medications}

PRINIVIL TABS 20 MG (LISINOPRIL) 1 po qd

Last Refill: \#30 × 2 : Carl Savem MD (08/27/2010)

HUMULIN INJ 70/30 (INSULIN REG \& ISOPHANE (HUMAN)) 20 units ac breakfast Last Refill: \#600 u x 0 : Carl Savem MD (08/27/2010)

\section{Directives}

Allergies and Adverse Reactions ( = critical)

OFFICE VISIT

History of Present Illness

Reason for visit: Routine follow up

Chief Complaint: No complaints

History

Diabetes Management

Hyperglycemic Symptoms

Polyuria: no

Review of Systems

General: denies fatigue, malaise, fever, weight loss

Eyes: denies blurring, diplopia, irritation, discharge

Ear/Nose/Throat: denies ear pain or discharge, nasal obstruction or discharge, sore

throat

Cardiovascular: denies chest pain, palpitations, paroxysmal nocturnal dyspnea,

orthopnea, edema Respiratory: denies coughing, wheezing, dyspnea, hemoptysis

Gastrointestinal: denies abdominal pain, dysphagia, nausea, vomiting, diarrhea

Vital Signs

Ht: 64 in. Wt: 140 lbs.T: 98.0 degF. T site: oral P: 72 R: 16 BP: 158/90

Physical Exam

General Appearance: well developed, well nourished, no acute distress

Eyes: conjunctiva and lids normal, PERRLA, EOMI, fundi WNL

Ears, Nose, Mouth, Throat: TM clear, nares clear, oral exam WN

Respiratory: clear to auscultation and percussion, respiratory effort normal

Cardiovascular: regular rate and rhythm, S1-S2, no murmur, rub or gallop, no bruits,

peripheral pulses normal and symmetric, no cyanosis, clubbing, edema or varicosities

Skin: clear, good turgor, color WNL, no rashes, lesions, or ulcerations

Plan

Medications:

HUMULIN INJ 70/30 20 u ac breakfast

PRINIVIL TABS 20 MG 1 qd

Treatment: Will have annual foot exam at next visit.

Figure 1: Shortened version of a public EHR ${ }^{1}$. Sections that we classify are highlighted in different colors.

medications have their own section, they also appear in the Plan section. Other issues that are not exposed in this example include: 1) Section order is not consistent across EHRs, 2) Headers may be missing, 3) Common features of headers (e.g. bold or colon) are not guaranteed to appear.

Prior approaches in EHR section prediction have been specific to one source and have required significant annotation effort. We propose to reduce the annotation burden by augmenting training data using sections in medical literature (journals, textbooks, web content). Section headers in medical literature are often much more consistent than in EHRs, allowing us to identify with high precision a large number of training example sections using a small number of simple patterns. And although the style and content of sections is different from EHRs, our hypothesis is that there is enough
Review of systems

a psychiatric review of systems may include screening questions directed at identifying or exploring co morbid psychiatric illnesses or issues e $g$ sigecaps mnemonic or phq 9 for depression generalized anxiety disorder 7 for anxiety digfast mnemonic for mania or specific questioning around psychoses or other psychiatric complaints a full review of systems should attempt to identify and list all of the relevant stressors that may be impacting a patient $s$ function and overall health

\section{Diagnosis}

ultrasound remains as one of the only effective ways of prenatally diagnosing larsen syndrome prenatal diagnosis is extremely important as it can help families prepare for the defects ultrasound can capture prenatal images of multiple joint dislocations abnormal positioning of legs and knees depressed nasal bridge prominent forehead and club feet these symptoms are all associated with larsen syndrome so they can be used to confirm that a fetus has the disorder arrival of an infant with several

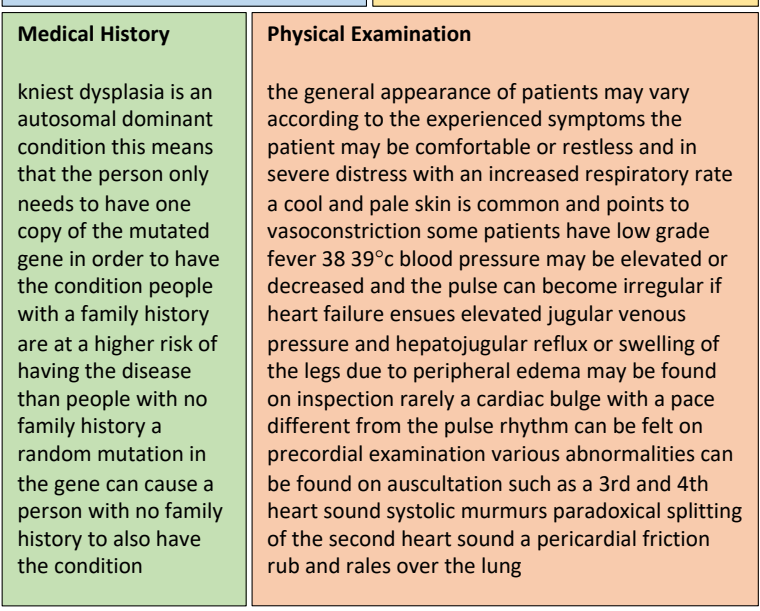

Figure 2: Sections from Wikipedia articles (Psychiatric history, Larsen syndrome, Kniest dysplasia, and Myocardial infarction diagnosis) that are similar to sections that occur in EHRs. Colors match sections types from Figure 1. The four headers map to the classes ROS, Find, $P M H$, and Exam.

similarity in language to help train models to detect sections in EHRs. Figure 2 shows paragraphs from Wikipedia articles whose sections are similar to those found in EHRs. The text tends to be more descriptive of the kinds of things that could be found in an EHR, but there is semantic overlap.

Our approach is not specific to one hospital or software system and uses data where labels are easy to infer without the need of a time-consuming annotation effort. Furthermore, the large amount of data allows us to explore state of the art neural classification approaches. Finally, training from medical literature can aid in identification of common sections in EHRs from different sources, making it possible to share EHR information more easily across medical facilities and insurance companies which may have access to EHRs from multiple providers.

Our work has two main contributions, demonstrating that augmenting automatically labeled 
data from medical literature with:

1. a small amount of labeled in-domain EHR training data significantly improves prediction in the EHR dataset.

2. labeled EHR data from a different source (out-of-domain) significantly improves the transferability of models trained when there is no labeled data in the in-domain EHR dataset.

\section{Related Work}

Prior work in EHR section prediction has focused on the following tasks:

1. Section detection: detecting the boundaries of sections; detecting section header text.

2. Section classification: assigning a class label to a section or to sentences (a section class label on a sentence indicates the section to which a sentence likely belongs).

Research may concentrate on section detection only (Ganesan and Subotin, 2014; Dai et al., 2015), section classification (with section boundaries assumed to be known) (Li et al., 2010; Haug et al., 2014) or both (Apostolova et al., 2009; Denny et al., 2009; Tepper et al., 2012). In this paper we focus on section-level classification and section classification at the sentence level.

Prior approaches to section prediction include Support Vector Machines leveraging features computed by bi-gram tf-idf vector representations (Apostolova et al., 2009), Hidden Markov Models (HMM) with sections regarded as part of a sequence (Li et al., 2010), Maximum Entropy Classifiers (Tepper et al., 2012), $\ell 1$-Regularized Logistic Regression (Ganesan and Subotin, 2014), Bayesian models using $\mathrm{N}$-gram features (Haug et al., 2014), and linear-chain Conditional Random Fields (CRF) to determine section headers (Dai et al., 2015). Most of these approaches rely heavily on hand-crafted features that are time consuming to develop and may not easily generalize across EHRs from different sources. To the best of our knowledge no prior work has explored deep learning or transfer learning. Given enough data, deep neural networks can extract useful features automatically. Some prior work is able to capture regularities in section ordering, either by using the Viterbi Algorithm (Ganesan and Subotin, 2014; Li et al., 2010) or beam search (Tepper et al., 2012) during the final section labeling phase, or by incorporating section ordering features (e.g. the class of the section preceding the section to be classified) into the model (Tepper et al., 2012). In this paper, since we mainly focus on transfer learning based on deep learning models, we predict labels for each section or sentence independently, leaving section sequence considerations to future work.

Two previous efforts have explored more than one dataset (Tepper et al., 2012; Ganesan and Subotin, 2014). Both evaluated domain adaptability and found significant reduction in performance across domains. The largest dataset, i2b2, has 13,962 expert-labeled sections (Dai et al., 2015). We use this dataset in our experiments as well. Because of the difficulty of annotating training data, some prior work adopts a semi-automated labeling approach (Apostolova et al., 2009; Denny et al., 2009; Li et al., 2010; Ganesan and Subotin, 2014) as we do for our medical literature dataset.

With the exception of the approaches using $\mathrm{i} 2 \mathrm{~b} 2$, all prior work uses proprietary datasets that are not available publicly making it difficult to compare to earlier approaches. We make our MedLit dataset as well as our extension of the labels for the i2b2 dataset available for research.

\section{Data}

We chose a set of 11 section class labels based on those used in the prior work discussed in Section 2 and on the most common sections from EHRs in our sources. The 11 classes are:

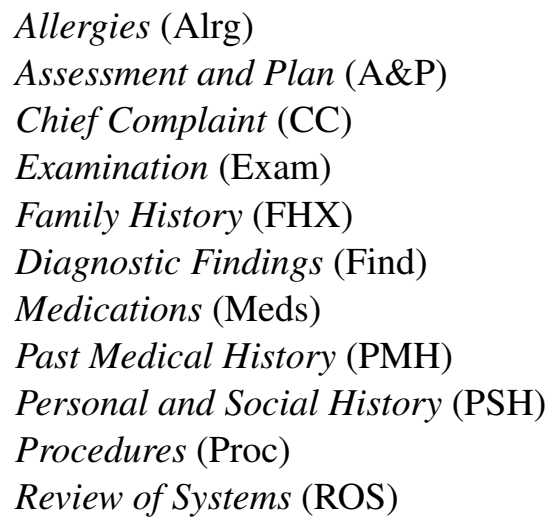

For our experiments we use three datasets: Medical Literature (MedLit), Cleveland Clinic EHRs (ClvC), and i2b2 EHRs (i2b2). 


\begin{tabular}{l|c|c} 
Source & \# Sections & \# Sentences \\
\hline Textbooks & 807 & 7,407 \\
Guidelines & 4,781 & 53,013 \\
Wikipedia & 2,658 & 24,830
\end{tabular}

Table 1: Sections and sentences in each MedLit source.

\subsection{Medical Literature (MedLit)}

The medical literature dataset consists of passages from textbooks, guidelines, and a subset of medically relevant Wikipedia articles ${ }^{2}$. The number of sentences per source type is shown in Table 1 . In total there are four sources in this dataset: Wikipedia and licensed content from DynaMed, Elsevier, and Wiley publishers.

To generate training data, we created a short list of common phrases for each of the $11 \mathrm{sec}-$ tion classes by looking at labels used in prior work as well as section headers labeled in our annotated EHR datasets. We extracted sections in the MedLit corpus whose headers (indicated by XML markup) matched these phrases. For example, any section whose header matched "Chief Complaint", "Reason for Admission", "CC", "Signs and Symptoms", "History of Present Illness", or "SUBJ" was extracted as a positive example of the Chief Complaint class $^{3}$. We experimented with partial phrase matches but ultimately decided to use exact match with stemming for higher precision on the common classes. For less frequent classes (e.g. Allergies, Family History, Personal and Social History), we allowed additional words to be present in the title. We make the 2,658 sections and labels for the Medical Wikipedia dataset available publicly for research purposes. Examples of sections and their headers are shown in Figure 2, with corresponding class labels indicated in the caption. In the discussion section (Section 5.3) we analyze the quality of the MedLit dataset as a cheaply labeled resource.

\subsection{I2b2 EHRs (i2b2)}

We use the i2b2 Risk Factors dataset (Stubbs and Uzuner, 2015; Stubbs et al., 2015) which was annotated for section header boundaries (Dai et al., 2015). The annotations do not indicate section

\footnotetext{
${ }^{2}$ Articles under the 'Clinical Medicine' category (https://en.wikipedia.org/wiki/Category: clinical_medicine).

${ }^{3}$ It is common for History of Present Illness to be its own section but it is often part of or interchangeable with Chief Complaint so we opted to combine these two sections.
}

\begin{tabular}{c|c|r|r|r} 
& Source & \# Sections & \# Sentences & Ratio \\
\hline Train & MedLit & 7042 & 72191 & 10 \\
& i2b2 & 1680 & 10093 & 6 \\
& ClvC & 294 & 3467 & 12 \\
Dev & MedLit & 1204 & 13059 & 11 \\
& i2b2 & 1591 & 9373 & 6 \\
& ClvC & 404 & 3282 & 8 \\
Test & i2b2 & 3098 & 19110 & 6 \\
& ClvC & 404 & 4046 & 10
\end{tabular}

Table 2: The number of sections and sentences as well as the ratio of sentences to sections in each of the three datasets for train, dev, and test sets.

class so we matched the headers to our 11 classes. We used partial matches for this data due to the variability of EHR header text. There were 743 unique headers in the Dai et al. (2015) dataset that map to our 11 headers.

\subsection{Cleveland Clinic EHRs (ClvC)}

The Cleveland Clinic dataset consists of 178 deidentified patient notes from 54 patients acquired through a research collaboration agreement with Cleveland Clinic. The notes were annotated by two medical students in prior work. Interannotator agreement was computed on the first 34 notes (containing 106 sections) annotated by both annotators. The $\kappa$ score was 0.86 for the sections and 0.80 at the note level. Due to the high agreement between the annotators, the remaining notes were annotated by one annotator each. We manually mapped the section class labels from those annotations to our 11.

\subsection{Data Splits}

The MedLit dataset was split $80 / 20$ by section for training and tuning. We do not test on MedLit as our goal is finding sections in EHRs. The ClvC EHR dataset was split by patient into $60 \%$ for training and tuning, and $40 \%$ for testing. For i2b2 (Stubbs and Uzuner, 2015; Stubbs et al., 2015) we use Set 1 and 2 for training and development, and the i2b2 Test Set for testing. The distribution of the train, development/tuning (dev), and test is shown in Table 2. MedLit is the largest dataset. It has more than twice as many sentences as i2b2 and 8 times as many as ClvC. For some of the classes (e.g. Allergies and Personal and Social History) MedLit data is harder to find. Procedures is the smallest category in $\mathrm{i} 2 \mathrm{~b} 2$ (102 sentences) and Allergies is the smallest category in $\mathrm{ClvC}$ with 
only 35 sentences in the training data.

\section{Method and Results}

Our work addresses the scenario of an EHR dataset (a target dataset) with little or no training data for section classification. We would like to measure how well models trained on a different EHR dataset and/or on medical literature (source datasets) can be transferred to classify the target dataset. We use each of the $\mathrm{ClvC}$ and $\mathrm{i} 2 \mathrm{~b} 2$ datasets in turn as target, with the other as source. We also experiment with using the MedLit dataset as source, both alone and together with data from a source EHR dataset. Specifically, for each target EHR dataset we compare the following models:

- $C l v C$ : train on labeled data from ClvC EHRs

- i2b2: train on labeled data from i2b2 EHRs

- MedLit: train on medical literature data only

- MedLit + TR ClvC: take the MedLit model, then continue to train (transfer) on labeled ClvC data

- MedLit TR i2b2: take the MedLit model, then continue to train (transfer) on labeled i2b2 data

In all cases we evaluate our model on our two test sets: $\mathrm{ClvC}$ and $\mathrm{i} 2 \mathrm{~b} 2$. The data used in the test set is considered the target, or in-domain dataset. The other dataset(s) are considered source, or outof-domain, for that experiment and will differ depending on the experiment. We consider the model that trains and tests on the same dataset to be an upper bound (UB). Given enough data ${ }^{4}$, we would expect this configuration to perform the best.

We show our results with two different approaches. The first is a Recurrent Neural Network (RNN) using Gated Recurrent Unit (GRU) cell (Cho et al., 2014) and attention mechanism (Bahdanau et al., 2015). GRU aims to solve the vanishing gradient problem revealed in standard RNNs. In our model, attention is used to generate a weighted sum of GRU cell outputs for each word in the input text, for predicting the classification label (rather than only using the output from the last cell). The motivation is to let the model focus on those words that are the most useful for prediction, especially for long input text.

\footnotetext{
${ }^{4}$ In the case of $\mathrm{ClvC}$ we do not have enough annotated data to train a model that would be considered a good upper bound
}

The weights are computed by the soft alignment scores between each of the outputs and the last output of the RNN.

The second approach is based on BERT (Devlin et al., 2019), a state-of-the-art language representation model that pre-trains bidirectional representations by jointly conditioning on both left and right context. Once pre-trained, a BERT model can be fine-tuned to specific tasks. In our setting, we take the output of the transformer for the first token in the input, i.e., the special [CLS] word embedding, as the representation of the input, which is then used for label prediction by feeding into a classification layer.

We also experimented with a Convolutional Neural Network text classification model (Kim, 2014) as well as traditional machine learning models (Naive Bayes and SVM) using n-gram features, but all performed worse than our GRU RNN and BERT.

For the GRU RNN, we use the Adam optimizer, a batch size of 32, dropout of 0.2 , and embedding size 300 . We experimented with other parameter values on the development set, but these worked best. We ran each model for 50 epochs-enough for the training loss to converge. For our BERT experiments we use a PyTorch implementation ${ }^{5}$ with the bert-base-uncased model. We use the default BERT parameters including the BERT Adam optimizer, a batch size of 32, dropout of 0.1 , and embedding size 768. All text is cut off to the first 128 word-pieces. We experimented with different numbers of epochs, and chose the model that performed best on the dev set (usually one tuned at 10 epochs or fewer). Statistical significance was computed using McNemar's test. We experimented with both section classification and sentence classification as described in the following subsections.

Our transfer setting follows the pre-training and fine-tuning approach: after training on the large source domain (MedLit), we continue to tune the model on a small amount of labeled data from the target domain. We follow this approach using our RNN as well as the BERT model where we first tune BERT to the MedLit data and then continue to tune that model on the EHR data. We also experimented with using subsets of the MedLit dataset, downsampling, and class balancing. These exper-

\footnotetext{
${ }^{5}$ https://github.com/huggingface/ pytorch-pretrained-BERT
} 


\begin{tabular}{|l|l|l|}
\hline Experiment & RNN F1 & BERT F1 \\
\hline \hline MedLit & 0.47 & 0.55 \\
\hline ClvC (UB) & $0.59^{+}$ & 0.89 \\
MedLit + TR ClvC & $\mathbf{0 . 7 8}^{*}$ & $\mathbf{0 . 9 0}$ \\
\hline i2b2 & 0.71 & 0.83 \\
MedLit + TR i2b2 & 0.65 & 0.78 \\
\hline
\end{tabular}

(a) Testing on ClvC

\begin{tabular}{|l|l|l|}
\hline Experiment & RNN F1 & BERT F1 \\
\hline \hline MedLit & 0.68 & 0.76 \\
\hline i2b2 (UB) & $\mathbf{0 . 9 5}$ & $\mathbf{0 . 9 9}$ \\
MedLit + TR i2b2 & 0.93 & $\mathbf{0 . 9 9}$ \\
\hline ClvC & $0.53^{+}$ & $0.84^{+}$ \\
MedLit + TR ClvC & $0.81^{*}$ & $0.92^{*}$ \\
\hline
\end{tabular}

(b) Testing on i2b2

Table 3: Section-Level results for testing on the (a) ClvC and (b) i2b2 EHRs. F1-scores are micro averages across all classes. The best results in each column are highlighted in bold. The results are grouped by MedLit, in-domain ((a) ClvC, (b) i2b2), and out-of-domain ((a) i2b2, (b) ClvC) training. * is significantly higher than + at $p<=.01$.

iments resulted in no significant change or a negative change to the results ${ }^{6}$, so we report results using the complete, raw MedLit dataset. We expect that the models learn which data sources are more useful.

\subsection{Section Classification}

Our first set of experiments follow the approach of prior work (Li et al., 2010; Haug et al., 2014) in classifying sections of the EHR. The section header is included in the data if it exists. As noted in prior work, this task is easier than sentence classification as it is common for the header to be discoverable with accuracy $>90 \%$ for most categories. We find similar trends in the $\mathrm{i} 2 \mathrm{~b} 2$ data with the upper bound (train on $\mathrm{i} 2 \mathrm{~b} 2$, test on $\mathrm{i} 2 \mathrm{~b} 2$ ) achieving an average $95 \%$ F-score with the RNN and near perfect F-score of $99 \%$ with BERT.

Results of experiments using the Cleveland Clinic (ClvC) test set as target are shown in Table 3a. The ClvC upper bound using the RNN (59\% average F-score) is much lower than is typical in prior work, underlining the difficulty of even section-level classification with small amounts of training data (294 sections). Using the RNN model trained on MedLit alone does quite poorly, but tuning on MedLit prior to training on $\mathrm{ClvC} \mathrm{im-}$ proves results very significantly over ClvC training alone (average F-score of 78\%). BERT improves the results significantly here with $89 \%$ Fscore, but the $90 \%$ F-score when tuning on the MedLit data is not significantly better. The bottom rows of the table show the scenario where no indomain (ClvC) data exists, so we train on out-ofdomain (i2b2) data. In this case, training on $i 2 b 2$ alone is better than pre-training first on MedLit. When there is enough EHR data (even from a dif-

\footnotetext{
${ }^{6}$ There is some change in the performance when using Wikipedia only, but not in the overall trends
}

ferent source), the MedLit data does not help.

Table $3 b$ shows results using the $i 2 b 2$ test set as target. With the large amount of in-domain i2b2 training data available, using MedLit does not help. The bottom rows of Table $3 \mathrm{~b}$ shows performance if we did not have $\mathrm{i} 2 \mathrm{~b} 2$ training data. Using the out-of-domain $(\mathrm{ClvC})$ training data performs poorly. Transferring MedLit with a very small amount of out-of-domain ( $\mathrm{ClvC})$ data significantly outperforms using just the $\mathrm{ClvC}$ data for both the RNN and BERT, improving average F-score from $53 \%$ to $81 \%$ for the RNN and from $84 \%$ to $92 \%$ for BERT.

\subsection{Sentence Classification}

Prior work on section-level classification assumes that the section segmentation and header are known. In practice, this is not always the case. It is common for headers to be missing or unclear. In our experiments, therefore, we don't assume to know where the header is and instead of trying to classify a section we classify all sentences in the EHR. For training data for this task we take the class label annotated on each section in our datasets and attach it to each sentence in the section. So each sentence in the section (including the header) is considered an instance of the class. The sentence predictions could be combined to provide section level boundaries. We discuss this further as future work in Section 6. In the MedLit training data, we exclude sentences that are too small (fewer than 15 characters) or too large (more than 400 characters). As with our section-level experiments (Section 4.1), we evaluate our performance with the $\mathrm{ClvC}$ and $\mathrm{i} 2 \mathrm{~b} 2$ test sets. We first tuned the MedLit model using the MedLit development set as described in Section 4. We found that with BERT, only one epoch was necessary, with more epochs resulting in over-fitting. 


\begin{tabular}{|l|l|l|}
\hline Experiment & RNN F1 & BERT F1 \\
\hline \hline MedLit & 0.30 & 0.37 \\
\hline ClvC (UB) & $0.61^{+}$ & $0.73^{+}$ \\
MedLit + TR ClvC & $\mathbf{0 . 6 2}^{*}$ & $\mathbf{0 . 7 4}^{*}$ \\
\hline i2b2 & 0.56 & 0.62 \\
MedLit + TR i2b2 & 0.53 & 0.60 \\
\hline
\end{tabular}

(a) Testing on ClvC

\begin{tabular}{|l|l|l|}
\hline Experiment & RNN F1 & BERT F1 \\
\hline \hline MedLit & 0.30 & 0.41 \\
\hline i2b2 (UB) & $0.64^{+}$ & $\mathbf{0 . 7 1}$ \\
MedLit + TR i2b2 & $\mathbf{0 . 6 5}^{*}$ & $\mathbf{0 . 7 1}$ \\
\hline ClvC & $0.48^{+}$ & $0.59^{+}$ \\
MedLit + TR ClvC & $0.52^{*}$ & $0.60^{*}$ \\
\hline
\end{tabular}

(b) Testing on i2b2

Table 4: Sentence-Level results for testing on the (a) ClvC and (b) i2b2 EHRs in Micro Avg F1-score. The best results in each column are highlighted in bold. The results are grouped by MedLit, in-domain ((a) ClvC, (b) i2b2) and out-of-domain ((a) i2b2, (b) ClvC) training. * is significantly higher than + at $p<=.01$.

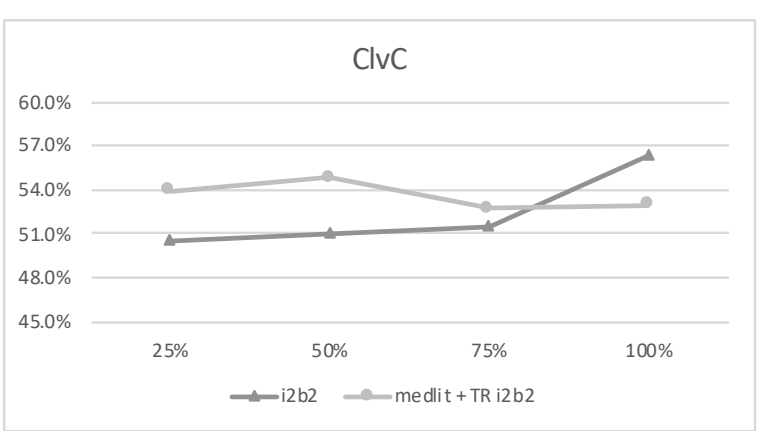

Figure 3: F-score trends of our RNN model training on smaller portions of the i2b2 data for the i2b2 and MedLit + TR i2b2 experiments and testing on ClvC

The results for testing on the $\mathrm{ClvC}$ data are shown in Table 4a. Although we call the configuration where we train and test on $\mathrm{ClvC}$ data the "upper bound", the MedLit + TR ClvC performs significantly $(p<=.01)$ better for the RNN $(62 \%$ vs $61 \%$ ) and BERT models (74\% vs $73 \%$ ). We believe that this is because the $\mathrm{ClvC}$ dataset is small so the upper bound is not achieved. On the other hand, because the $\mathrm{i} 2 \mathrm{~b} 2$ training dataset is large, training on the MedLit data first does not provide a significant improvement with either model. We analyzed this result further by experimenting on training with subsets $(25 \%, 50 \%$, and $75 \%)$ of the $\mathrm{i} 2 \mathrm{~b} 2$ training data and testing on $\mathrm{ClvC}$ for the i2b2 and MedLit + TR i2b2 experiments. Figure 3 shows that with less out-of-domain EHR training data, transfer learning does help significantly ( $p$ value $<=.01$ ) in all cases for the RNN model. In fact, there is no significant difference between using $25 \%$ or $100 \%$ of the $i 2 \mathrm{~b} 2$ training data in the MedLit + TR i2b2 experiment.

The sentence-level results for testing with $\mathrm{i} 2 \mathrm{~b} 2$ as target are shown in Table 4b. Using the MedLit data in the RNN provides a significant improvement $(65 \%$ vs $64 \%)$. However, there is no differ-

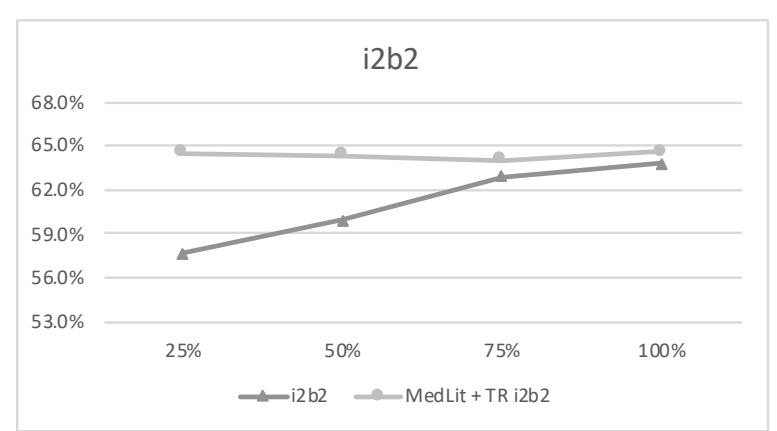

Figure 4: F-score trends of our RNN model training on smaller portions of the $i 2 b 2$ data for the $i 2 b 2$ and MedLit + TR i2b2 experiments and testing on i2b2

ence in the BERT models, likely because of the a large amount of $i 2 b 2$ training data. Here again, we explored how having a smaller subset of the indomain training data would impact the results. We found that running the MedLit + TR i2b2 experiment with just $25 \%$ of the i2b2 dataset with the RNN model performs as well (no significant difference) as training on the full i2b2 dataset (Figure 4), and $12 \%$ better than the model using $25 \%$ of the $\mathrm{i} 2 \mathrm{~b} 2$ data alone. In contrast, when using the BERT model, MedLit + TR i2b2 only performs better than i2b2 alone if less than $25 \%$ of the training data is available. This indicates that BERT can compensate for the lack of data while the RNN cannot, and MedLit is needed to improve the model. In the scenario where no in-domain training data is available we show that MedLit + TR ClvC does significantly better than just ClvC when testing on $\mathrm{i} 2 \mathrm{~b} 2$ for the RNN model (52\% vs $48 \%$ ) and BERT (60\% vs 59\%). We discuss these results further in the next section 


\begin{tabular}{|l|l|l|l|}
\hline Experiment & F1 @ & F1@2 & F1@ 3 \\
\hline RNN ClvC & 0.48 & 0.66 & 0.75 \\
RNN MedLit + TR ClvC & 0.52 & 0.69 & 0.79 \\
BERT ClvC & 0.59 & 0.75 & 0.82 \\
BERT MedLit + TR ClvC & 0.60 & 0.75 & 0.83 \\
\hline
\end{tabular}

Table 5: Average F1 score for training on ClvC and testing on $\mathrm{i} 2 \mathrm{~b} 2$ when examining the top 3 predictions.

\section{Discussion}

\subsection{How much do out-of-domain EHRs help?}

One of our most interesting results using the full datasets is the improvement we are able to achieve training with a small amount of ClvC (out-ofdomain) EHR data and testing on $\mathrm{i} 2 \mathrm{~b} 2$ as the target domain (the last two rows of Table $3 \mathrm{~b}$ and Table 4b). All of these results on the sentence level are quite low due to similar text that may be found in multiple sections. For example, the text found for Chief Complaint and Past Medical History can be quite similar with the only difference being when the problems occurred. To determine if this kind of misclassification was common we looked at our performance based on whether the correct class was in the top 1, 2, or 3 predictions (F1@3). Both ClvC and MedLit+TR ClvC showed large improvements for the RNN and BERT models as shown in Table 5 with roughly a 27 point improvement for the RNN models and a 22 point improvement for the BERT models. We analyzed the confusion matrix and the most common misclassifications were to the majority MedLit and ClvC classes (Assessment and Plan and Chief Complaint).

\subsection{Do models impact sections differently?}

We also compared performance on the individual classes when transferring from MedLit. To do this, we explored the training on $\mathrm{i} 2 \mathrm{~b} 2$ and testing on BERT i2b2, and BERT MedLit + TR i2b2 experiments (See the middle rows in Table $4 \mathrm{~b}$ ) where both models performed the same. We found the BERT i2b2 performed better for Allergies (6.8\%), but BERT MedLit + TR i2b2 performed better for Family History (5.8\%), and Procedures (7.9\%). This suggests that it may be worth exploring an ensemble approach.

\subsection{What is the quality of the MedLit data?}

How well our approach performs on EHR section classification relies on the MedLit data. The
MedLit dataset is cheaply labeled data of a different genre than the target data, so we would not expect the MedLit model to perform particularly well on its own on EHR data. To judge the quality of the MedLit-trained model in its own genre, we analyzed its performance on the dev set made up solely of medical literature. The best average F-score was $65.2 \%$ using the RNN and $71.2 \%$ for BERT on the sentence classification task. In particular, we found that Assessment and Plan performs quite poorly. We examined the confusion matrix and the predictions are distributed among the other classes indicating that it is more vague. Looking to see if the correct label is at the secondor third-highest scoring predictions, we find the F1@3 is $88.9 \%$ for the RNN model and $92.6 \%$ for the BERT model. This is significantly better, but still shows that it is weakly labeled data. We also analyzed the performance from our different medical literature sources. This analysis caused us to drop one of our initial guideline sources because it had few useful labels. The two relevant labels it did have were easy to predict correctly causing a bias toward the source. Finally, we inspected the text for the section headers. There were 66 unique headers in the medical literature dev set ranging over the 11 classes. "Physical", which maps to the Examination class was the most common section header and was predicted correctly $74 \%$ and $79 \%$ of the time for the RNN and BERT models respectively. Some other good strings include "History of Present Illness" and "signs and symptoms" for the Chief Complaint class and "medication" for the Medications class. Our Review of Systems headers included common subsections found in this area of the EHR such as "eye", "neurology", and "genitourinary". Some of these did not perform as well, probably because they are likely to appear in many different sections. We also analyzed the confusion matrix to determine the common misclassifications. In most cases the common misclassifications were to Chief Complaint, the majority class. We also found that Past Medical History was often incorrectly labeled as Medications. We found this analysis to be consistent across both models indicating that the data is not model dependent.

\section{Conclusion}

In this paper we describe a novel approach to classifying sections in Electronic Health Records 
when a limited amount of in-domain training data is available. We present a new dataset for EHR section prediction from Medical Literature, of which the Wikipedia part is available to the public for research purposes. We show that combining a very small amount of in-domain EHR data with a large amount of automatically labeled, out-of-domain, out-of-genre Medical Literature data can perform as well as using a large amount of in-domain EHR data at the section and sentence level. We also show that combining out-of-domain, out-of-genre Medical Literature data with out-of-domain EHRs can provide significant improvement over using just out-ofdomain EHRs at the section and sentence level, depending on training data size. These results indicate that even though the data in Medical Literature is very different in style, the content can bridge between the domain-specific vocabularies of different EHR systems. We show that our approach can be used to achieve good results on new unseen EHR datasets with minimal or even no training data. In the future we would also like to explore using both $\mathrm{i} 2 \mathrm{~b} 2$ and $\mathrm{ClvC}$ together to see if a multi-task learning approach would provide additional improvements. In addition to BERT, we also briefly explored BioBERT (Lee et al., 2019), a BERT model pre-trained on a medical corpus. In our initial experiments BioBERT performed worse than BERT, but we would like to explore this further.

Finally, in this work we focus on individual sentence and section classification and show that we can achieve improvements in this regard. In addition, we could also exploit the structure of the document to provide additional improvements. The structure often follows a pattern in EHRs (for example, Chief Complaint tends to be the first section). Prior work has looked at CRFs and HMMs (Li et al., 2010; Dai et al., 2015) to exploit this property. We would like to explore whether we can improve our model by combining it with a model that takes into account trends at the document level. Using LSTM-CRFs (Lample et al., 2016; Huang et al., 2015) as a second level with BERT as pre-training on our model may provide such an improvement.

\section{Acknowledgments}

We thank the physicians and IT staff at Cleveland Clinic who provided de-identified EMRs under an
IRB protocol and the students from New York Medical College and SUNY Downstate Medical Center for their enthusiastic and dedicated annotation work.

\section{References}

Emilia Apostolova, David S Channin, Dina DemnerFushman, Jacob Furst, Steven Lytinen, and Daniela Raicu. 2009. Automatic segmentation of clinical texts. In Engineering in Medicine and Biology Society, 2009. EMBC 2009. Annual International Conference of the IEEE, pages 5905-5908. IEEE.

Dzmitry Bahdanau, Kyunghyun Cho, and Yoshua Bengio. 2015. Neural machine translation by jointly learning to align and translate. In 3rd International Conference on Learning Representations, ICLR 2015, San Diego, CA, USA, May 7-9, 2015, Conference Track Proceedings.

Kyunghyun Cho, Bart van Merrienboer, Caglar Gulcehre, Dzmitry Bahdanau, Fethi Bougares, Holger Schwenk, and Yoshua Bengio. 2014. Learning phrase representations using rnn encoder - decoder for statistical machine translation. In Proceedings of the 2014 Conference on Empirical Methods in Natural Language Processing (EMNLP), pages 17241734.

Hongjie Dai, Syed Abdul Shabbir, Chih-Wei Chen, and Chieh-Chen Wu. 2015. Recognition and evaluation of clinical section headings in clinical documents using token-based formulation with conditional random fields. BioMed Research International, 2015.

Joshua C. Denny, Anderson Spickard, III, Kevin B. Johnson, Neeraja B. Peterson, Josh F. Peterson, and Randolph A. Miller. 2009. Evaluation of a method to identify and categorize section headers in clinical documents. Journal of the American Medical Informatics Association, 16(6):806-815.

Jacob Devlin, Ming-Wei Chang, Kenton Lee, and Kristina Toutanova. 2019. BERT: Pre-training of deep bidirectional transformers for language understanding. In Proceedings of the 2019 Conference of the North American Chapter of the Association for Computational Linguistics: Human Language Technologies, Volume 1 (Long and Short Papers), pages 4171-4186, Minneapolis, Minnesota. Association for Computational Linguistics.

K. Ganesan and M. Subotin. 2014. A general supervised approach to segmentation of clinical texts. In 2014 IEEE International Conference on Big Data (Big Data), pages 33-40.

Peter J. Haug, Xinzi Wu, Jeffrey P. Ferraro, Guergana Savova, Stanley M. Huff, and Christopher G. Chute. 2014. Developing a section labeler for clinical documents. In AMIA 2014, American Medical Informatics Association Annual Symposium, Washington, DC, USA, November 15-19, 2014. 
Zhiheng Huang, Wei Xu, and Kai Yu. 2015. Bidirectional LSTM-CRF models for sequence tagging. CoRR, abs/1508.01991.

Yoon Kim. 2014. Convolutional neural networks for sentence classification. In Proceedings of the 2014 Conference on Empirical Methods in Natural Language Processing (EMNLP), pages 1746-1751, Doha, Qatar. Association for Computational Linguistics.

Guillaume Lample, Miguel Ballesteros, Sandeep Subramanian, Kazuya Kawakami, and Chris Dyer. 2016. Neural architectures for named entity recognition. In Proceedings of the 2016 Conference of the North American Chapter of the Association for Computational Linguistics: Human Language Technologies, pages 260-270. Association for Computational Linguistics.

Jinhyuk Lee, Wonjin Yoon, Sungdong Kim, Donghyeon Kim, Sunkyu Kim, Chan Ho So, and Jaewoo Kang. 2019. Biobert: a pre-trained biomedical language representation model for biomedical text mining. CoRR, abs/1901.08746.

Ying Li, Sharon Lipsky Gorman, and Noémie Elhadad. 2010. Section classification in clinical notes using supervised hidden markov model. In Proceedings of the 1st ACM International Health Informatics Symposium, IHI '10, pages 744-750, New York, NY, USA. ACM

Benjamin Shickel, Patrick Tighe, Azra Bihorac, and Parisa Rashidi. 2017. Deep EHR: A survey of recent advances on deep learning techniques for electronic health record (EHR) analysis. CoRR, abs/1706.03446.

Amber Stubbs, Christopher Kotfila, Hua Xu, and Özlem Uzuner. 2015. Identifying risk factors for heart disease over time: Overview of 2014 i2b2/uthealth shared task track 2. Journal of Biomedical Informatics, 58:S67 - S77. Proceedings of the 2014 i2b2/UTHealth Shared-Tasks and Workshop on Challenges in Natural Language Processing for Clinical Data.

Amber Stubbs and Özlem Uzuner. 2015. Annotating risk factors for heart disease in clinical narratives for diabetic patients. Journal of Biomedical Informatics, 58:S78 - S91. Proceedings of the 2014 i2b2/UTHealth Shared-Tasks and Workshop on Challenges in Natural Language Processing for Clinical Data.

Michael Tepper, Daniel Capurro, Fei Xia, Lucy Vanderwende, and Meliha Yetisgen-Yildiz. 2012. Statistical section segmentation in free-text clinical records. In Proceedings of the Eighth International Conference on Language Resources and Evaluation (LREC-2012). European Language Resources Association (ELRA). 